\title{
Architetture difensive nelle valli dello Ziz e del Todhra in Marocco
}

\author{
Defensive architecture in the Ziz and Todhra valleys in Morocco
}

\author{
Marinella Arena ${ }^{a}$, Paola Raffa ${ }^{\text {b }}$ \\ Università degli Studi Mediterranea, Reggio Calabria, Italy \\ a marinella.arena@unirc.it; ${ }^{\mathrm{b}}$ paola.raffa@unirc.it
}

\begin{abstract}
The earthen architecture of the Todhra and Ziz Valleys in Southern Morocco takes us back to the basic and archetypal forms of building in the Mediterranean. Architectural typology and language together form a cultural background that is strongly rooted in the territory and its inhabitants: the Berbers. The architectures, fragile and in constant decay, represent a treatise of living architecture in which the shapes, proportions and decorations are repeated over time with continuity.

This research tries to verify, with data coming from direct and instrumental surveys, the quality and diffusion of the architectures that dot the valleys of the Todhra and the Ziz which, at same time, host the population and defend the most precious asset: water. Along the valleys, united by the same language, we find: igherm, fortified citadels; tighremt, fortress houses.
\end{abstract}

Keywords: Survey, earthen architecture, tighremt, igherm.

\section{Introduzione}

Nelle Valli dello Ziz e del Todhra, l'acqua costruisce un paesaggio fatto di elementi essenziali: il corso d'acqua, l'oasi, l'architettura. L'intero sistema è definito da una serie di elementi che intrattengono strette relazioni di equilibrio tra l'ambiente costruito (architettura e oasi) e l'ambiente naturale (sorgenti d'acqua e deserto). L'alterazione di uno degli elementi compromette la stabilità dell'intero sistema. Le architetture in terra ci riportano alle forme basilari e archetipe del costruire. Tipologia e linguaggio architettonico configurano insieme un bagaglio culturale fortemente radicato nel territorio e nei suoi abitanti: i Berberi (Fig. 2). Il modello di insediamento nella configurazione di igherm e tighremt ${ }^{1}$ è riconoscibile e chiaro: l'impianto urbano, la distribuzione dello spazio, la morfologia, la tipologia delle abitazioni mettono in evidenza la ripetizione di forme ed elementi. Sono ele- menti fragili e in costante disfacimento, rappresentano un trattato vivente in cui le forme, le proporzioni e i decori sono reiterati nel tempo con continuità. L'individuazione di igherm e tighremt, la catalogazione per categorie formali e funzionali, la scelta degli esempi da rilevare, sono state le prime operazioni che hanno messo in evidenza la quantità e la qualità dell'architettura. Il disegno di sezioni territoriali ha reso possibile comprendere il rapporto che esiste tra il villaggio, la casa, i giardini dell'oasi, i canali di irrigazione e il corso d'acqua (Figg. 3 e 4). Il disegno ha rivelato anche lo stato e la consistenza dell'architettura, le parti mancanti e quelle che ancora resistono al tempo. Il rilievo di igherm e tighremt delle valli del Todhra e dello Ziz è stato eseguito nel $2013^{2}$ nell' ambito di una campagna rilevamento che ha coinvolto le quattro principali valli dell'Alto Atlante maroc- 
chino. Questa ricerca prova a verificare, con dati provenienti da rilievi diretti e strumentali, la qualità e la diffusione delle architetture che punteggiano le valli del Todhra e dello Ziz e che sono deputate sì ad accogliere la popolazione ma anche a difendere il bene più prezioso: l'acqua. Lungo le valli, accomunati dallo stesso linguaggio, troviamo: igherm, cittadelle fortificate, e tighremt, case fortezza.

La metodologia di studio indaga il territorio e l'architettura che, nella sua conformazione tipomorfologica, è solo una componente del complesso sistema insediativo.

La ricerca, ancora in corso, è strutturata in quattro fasi: 1) Produzione di una mappa con relative catalogazioni e localizzazioni di igherm e tighremt, nella valle del Todhra e dello Ziz; 2) Elaborazione di rilievi diretti e strumentali per la documentazione del patrimonio, in realtà molto esteso e difficile da raccontare nella sua interezza; 3) Rappresentazione dell' architettura e le sue relazioni con il territorio; 4) Analisi delle architetture rilevate e individuazione degli elementi ricorrenti tipologici e morfologici.

Questo studio prova a sviluppare un sistema di analisi aperto, utile alla documentazione, alla comprensione e alla messa in valore di questo immenso patrimonio, architettonico umano e sociale.

\section{Igherm}

Gli igherm delle Valli dello Ziz e del Todhra, così come quelle della Valle del Draa, si possono suddividere in due grandi categorie morfologiche: quelli a schema regolare, probabilmente $\mathrm{i}$ più antichi, e quelli con pianta organica poco definita, che si sono sviluppati per aggregazione attorno ad un tighremt. L'igherm è un villaggio circondato da mura difensive con alte torri di sorveglianza (Fig. 5). Le mura di cinta sono l'elemento di difesa, generano il recinto, il limite tra lo spazio dell'abitato, lo spazio dell'oasi e lo spazio assoluto del deserto.

L'altezza e lo spessore del muro dipendono dalla lunghezza, dall'epoca della costruzione, dalla morfologia del terreno. Il perimetro del muro su ogni lato è ripartito ad intervalli regolari dalle torri. Le torri, a differenza di quelle delle tighremt, sono tozze e robuste, la verticalità è enfatizzata dalla proporzione con il muro dal quale sporgono per circa il doppio dell'altezza.

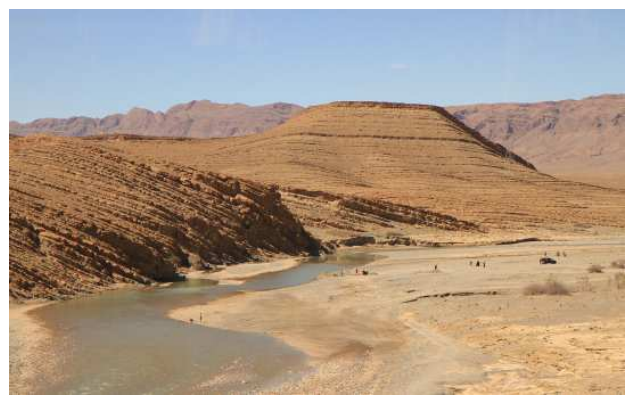

Fig. 1. Ziz Valley.

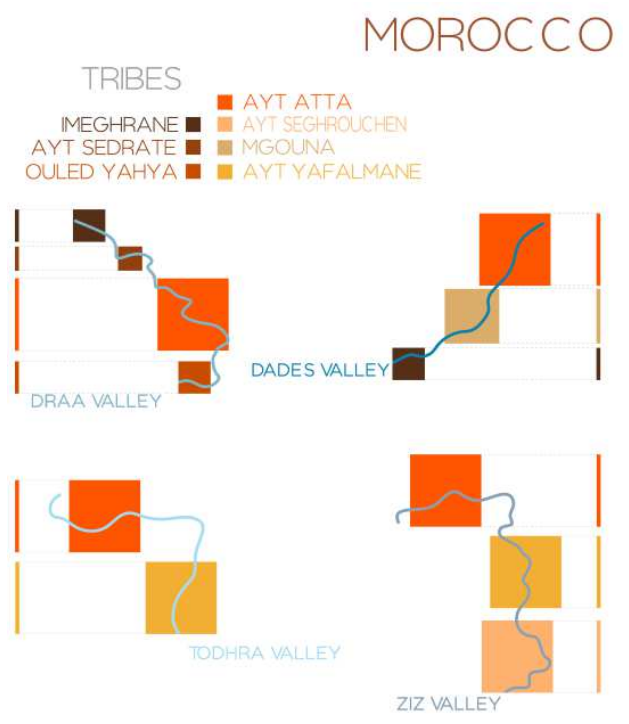

Fig. 2. Alto Atlante. Le tribù che abitano le valli dei quattro principali fiumi.

La torre è realizzata con blocchi di pisè nella parte bassa, e blocchi più piccoli in adobe, che consentono paramenti decorativi nella sommità. Una porta principale, imponente e ornata, segna l'ingresso al villaggio e la direzione prevalente dell'impianto urbano. 


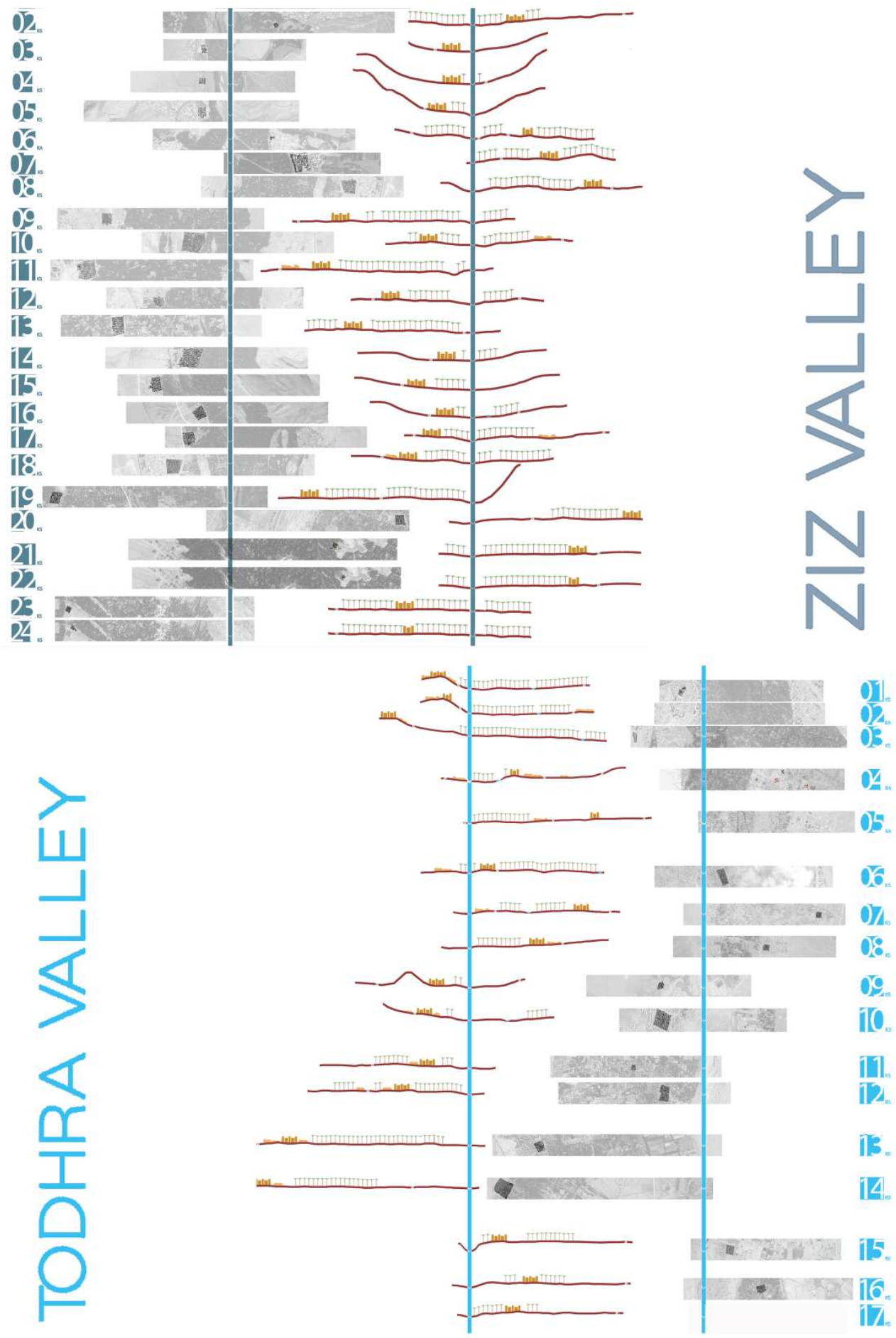

Figg. 3 e 4. Ziz Valley e Todhra Valley. Localizzazione di tighremt e igherm. 
Sia inserita nelle mura perimetrali, sia edificata all'esterno, l'entrata è una delle invarianti peculiari dell'insediamento. una delle invarianti peculiari dell'insediamento. Un grande corpo sporge dal sistema murario con una porta ad arco spesso preceduto da un muro ad L. Il sistema d'ingresso è un ambiente a pianta rettangolare suddiviso in due parti: da un lato il portico con arcate e panche rialzate, dall'altro la strada che conduce all'interno. Panche in muratura, giare per l'acqua ed il focolare per il tè, l'entrata assume la funzione di luogo d'incontro in cui si riunisce l'assemblea dei notabili ed in cui vengono ospitati viaggiatori e mercanti. Una seconda porta immette in un grande spazio aperto, in cui si svolgono le attività comunitarie, il mercato, le assemblee e le feste popolari. Qui si trova il recinto per gli animali, il deposito comune dei cereali, la cisterna dell'acqua, l'alloggio del guardiano. Da questo spazio aperto si diparte un'unica strada. La strada principale, ampia e retta, che suddivide i quartieri residenziali. In prossimità dell'ingresso e della piazza sono ubicati piccole botteghe artigianali, il panificio, il mercato, la moschea. All'esterno delle mura il cimitero con il mausoleo del santo protettore del villaggio, e nelle vicinanze un grande spazio libero per l'accampamento delle carovane di nomadi nei giorni di mercato. Intorno i terrazzamenti agricoli che ripetono in maniera speculare ed ordinata la ripartizione urbana. All'interno degli igherm, è sempre presente almeno una moschea, un complesso di ambienti che comprende una sala di preghiera, le sale per l'abluzione con la cisterna e le vasche, un focolare per riscaldare l'acqua, una scala per raggiungere la terrazza da dove il muezzin richiama alla preghiera. Le strade interne all'igherm sono lo spazio tra le abitazioni, non hanno altra funzione che quella della distribuzione dei quartieri, garantiscono la privacy e la sicurezza, quelle che immettono nei quartiere sono chiuse con porte. La struttura viaria è minima. Le strade sono strette e coperte dai piani superiori delle case, che coprendole creano zone d'ombra. Le abitazioni si dispongono le une accanto alle atre semplicemente accostate. La regola della prossimità assolve l'imperativo dell'occupazione del minor spazio possibile. I quartieri risultano nettamente separati tra loro ed organizzati in gerarchie etniche. L'abitazione nell'igherm è ancora un modello unico che si ripete nel tipo della casa corte. Le abitazioni si sviluppano in altezza. Al piano terra l'ingresso è un vano che distribuisce la scala, che porta ai piani superiori, e gli ambienti di servizio. Una stanza centrale è destinata al ricovero degli animali e degli attrezzi di lavoro, i vani intorno sono i magazzini per la conserva degli alimenti. Il primo livello si distribuisce attorno ad una corte circondata da una galleria con arcate a doppia o tripla altezza. Il foro che fuoriesce sulla terrazza è protetto da una griglia di legno o di ferro. Da qui entra la luce tenue che protegge dal caldo gli ambienti della casa. Il primo livello è ancora un piano di servizio, la zona per cucinare con arredi ricavati nei muri perimetrali ed il focolare modellato dal pavimento. Il secondo livello è la zona di soggiorno, gli ambienti si affacciano sulla corte centrale. L'ultimo livello è la terrazza. È uno spazio fondamentale in tutte le abitazioni. Anche qui sono presenti una o due stanze chiuse. Le tipologie rilevate presentano due, quatto o sei pilastri centrali, a seconda della grandezza della casa. L'utilizzo dell'adobe consente di organizzare motivi decorativi geometrici nella corte, all'interno delle stanze le pareti sono trattate con pitture colorate.

\section{Tighremt}

Le valli dei grandi fiumi dell'Alto Atlante marocchino sono un microcosmo ricco di acqua, vegetazione, vita, commercio, protetto dai venti caldi del Sahara e dalle incursioni nemiche dalle alture dello Jebel Saghro o dello Jebel Tadrat.I fiumi della regione, Draa, Dades, Todhra e Ziz, hanno modellato il territorio scavando gole profonde, in prossimità della foce, e disegnando grandi distese sabbiose, fertilissime, ai bordi del deserto. Le oasi, dense di palme e frutteti, saturano il letto del fiume coprendolo con un manto verdissimo. Igherm e tighremt punteggiano il paesaggio, hanno il colore della terra e sorgono in simbiosi con l'ambiente: modulano forma ed estensione in accordo al contesto. I tighremt sono case rurali fortificate. Accolgono la popolazione ma sono un baluardo contro gli scontri 

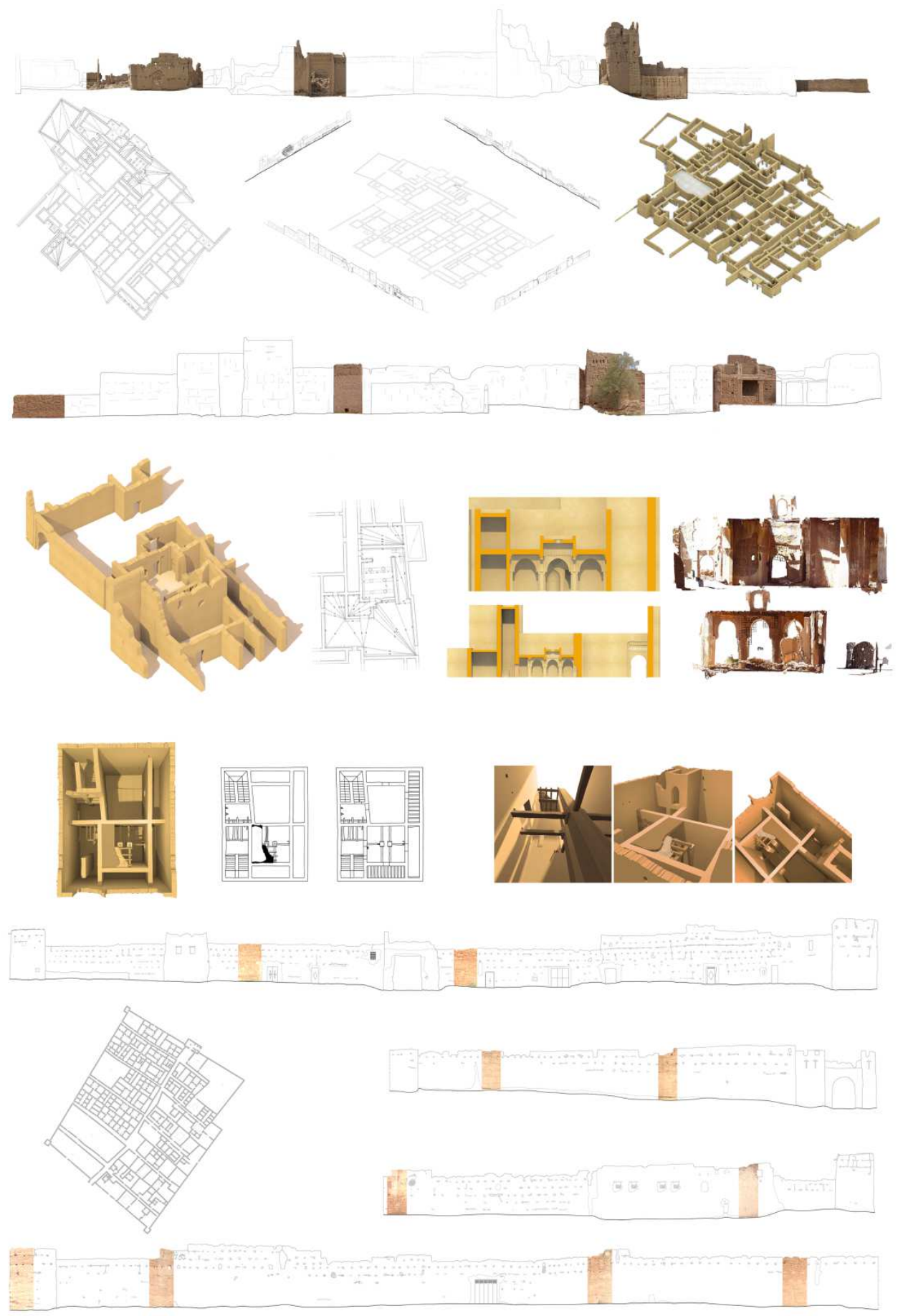

Fig. 4. Igherm della Ziz Valley. 


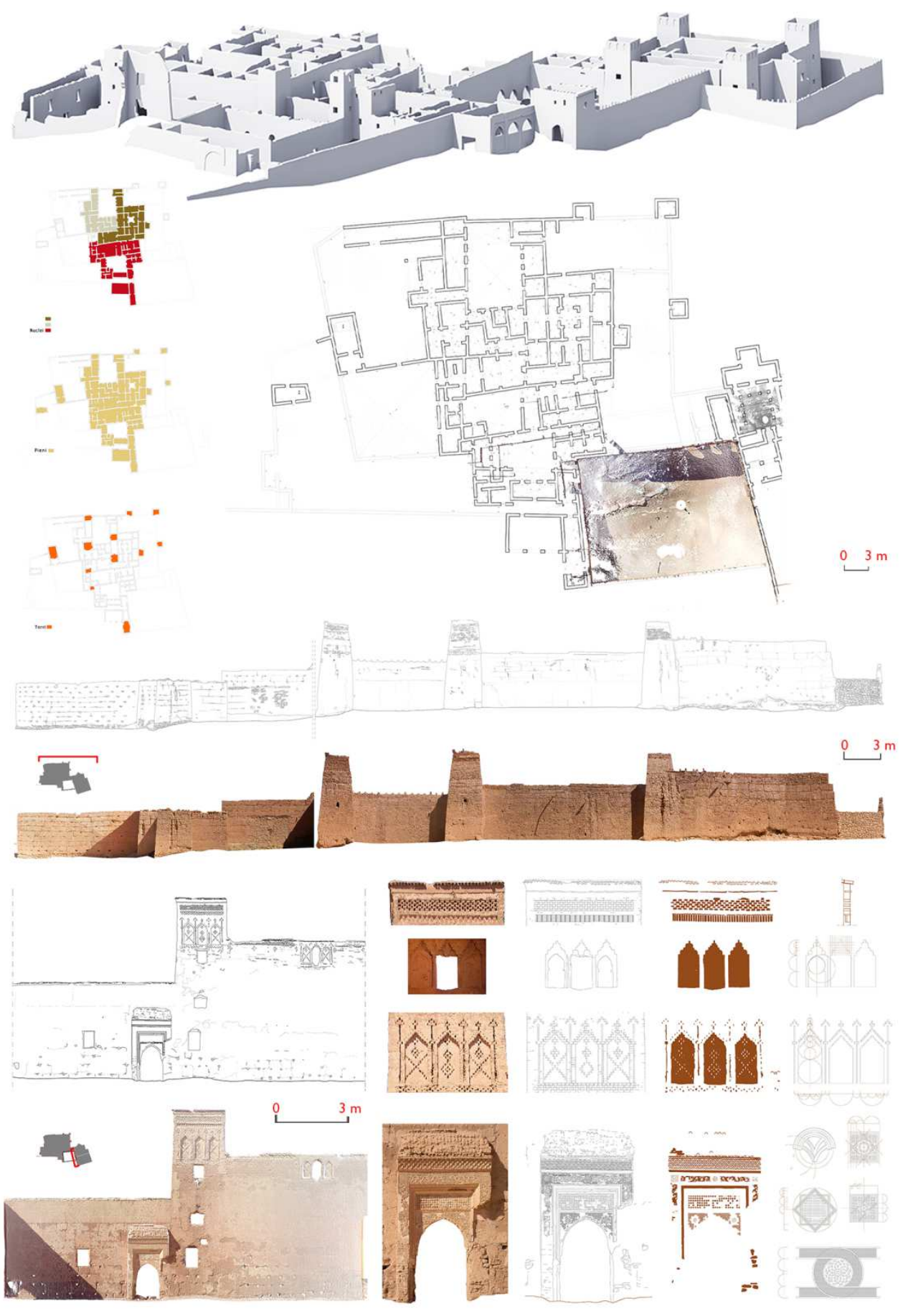

Fig. 5. Todhra Valley. Tinghir. Kasbah Pasha. 
tribali per il dominio dell'acqua o contro le incursioni delle tribù arabe nomadi. Lungo le valli dello Ziz e del Todhra troviamo sostanzialmente tre tipi di tighremt: quelli isolati; $\mathrm{i}$ grandi tighremt (kasbah); quelli che sorgono all'interno degli igherm. Questi ultimi, vere a proprie case dominanti, sono talvolta al centro dello sviluppo degli igherm. I tighremt isolati sorgono spesso dentro l'oasi sul terreno pianeggiante mentre i grandi tighremt, isolati, sono posti su alture rocciose, che prospettano sull'oasi, sono più estesi e presentano diverse corti, ampie, decorate con arcate e stucchi. Sono delle vere e proprie kasbah che devono dimostrare la forza e la presenza sul territorio del Cadì (Fig. 6). La tipologia dei tighremt è molto semplice: una pianta quadrata limitata da alti muri rinforzati da torrette quadrate. La disposizione interna prevede al primo livello gli ambienti destinati alla conservazione del grano "ahano", le stalle e naturalmente le scale che conducono al piano superiore. Al primo livello una piccola corte, solitamente coperta e molto piccola è il cuore della casa. Attorno ad essa ci sono piccole stanze per la famiglia utilizzate durate i mesi estivi. Al piano superiore e sulla terrazza altri piccoli ambienti sono utilizzati durante i mesi invernali. La corte centrale, taddawārit, ha la funzione di favorire la ventilazione e l'eliminazione dell' aria calda. La terrazza è circondata da alte mura per garantire l'intimità. La planimetria di un tighremt è molto semplice: alla pianta quadrata si affiancano quattro torrette d'angolo che hanno la duplice funzione di irrigidire i muri perimetrali e di difendere l'accesso al tighremt. Nelle kasbah, le dimore dei cadì o dei pasha, le corti sono più grandi e maggiormente decorate, mentre la tecnica costruttiva si mantiene inalterata. Infatti la parte basamentale dei muri è realizzata in grandi blocchi di pisè, llōh, larghi $1,2 \mathrm{~m}$ alti $0,8 \mathrm{~m}$ e profondi $0,6 \mathrm{~m}$, mentre la parte superiore è realizzata con mattoni interra cruda ottob che spesso sono disposti a creare piccole feritoie, per la ventilazione e il controllo delle mura perimetrali, isirr. I disegni creati dai mattoni sulle facciate interne ed esterne dei tighremt, lassserift, sono i vessilli della cultura tamazigh, berbera, e testimoniano l'appartenenza ad una tribù o ad uno specifico nucleo familiare.
Le architetture di questa regione sono progettate per resistere agli aggressori, al caldo ma temono l'abbandono. I aggressori e al caldo ma temono l'abbandono. I materiali semplici che le connotano, il pisè e i mattoni in terra cruda e lo speciale intonaco che impermeabilizza le terrazze e i muri perimetrali, tamassalt, hanno continua necessità di manutenzione. Le piogge, rare, sono spesso violente e i fiumi ricchi di acqua occasionalmente aumentano la loro portata distruggendo colture e abitazioni. Spesso i tighremt presenti nella valle del Todhra o dello Ziz sono disabitati, l'assenza di manutenzione lascia infiltrare l'acqua e il degrado avviene in pochissimo tempo. Le operazioni di rilievo di questa architetture sono rese difficili per la presenza in situ di detriti, per i crolli e la irregolarità delle murature. La campagna di rilevamento condotta nello Ziz e nel Todhra ha previsto, dopo una fase di catalogazione e di analisi del rapporto con il sito, alcuni rilievi puntuali di igherm e tighremt. In questo testo si riporta il caso della cosiddetta "Kasbah Pasha" che sorge in località Tinghir lungo le sponde del Todhra. Il tighremt è stato rilevato ibridando rilievo strumentale con rilievo diretto. Il rilievo strumentale ha consentito di raccogliere informazioni dettagliate sulla morfologia dei decori e sulla qualità della tessitura muraria. Il rilievo diretto invece ha consentito il riconoscimento dei singoli ambienti e la predisposizione di sezioni orizzontali esemplificative della struttura tipologica della kasbah.

\section{Conclusioni}

La ricerca è ancora in corso, come già accennato, ma l'esperienza del rilievo strumentale, unito a quello diretto, ha permesso di raccogliere dati sufficienti per alcune riflessioni. La prima è relativa alla catalogazione e sistematizzazione della architetture presenti nelle valli: sono necessarie alcune premesse sulla forma, la tipologia e lo stato di conservazione delle architetture catalogate. (Questa procedura, ad esempio, è stata applicata ai tighremt e igherm del Draa). La seconda riflessione riguarda la comunicazione delle informazioni raccolte. Questa oscilla, volutamente, fra il rigore astratto del segno stilizzato e il disegno a mano libera. La combinazione di questi due elementi potenzia, a nostro 
avviso, la qualità delle trame murarie e dei tessuti urbani.

\section{Note}

${ }^{1}$ I termini ighrem e tighremt, prorpi della lingua berbera, hanno un corrispondente arabo più conosciuto: ksar e kasbah.
${ }^{2}$ Nella valle dello Ziz, sono stati effettuati rilievi strumentali e diretti degli ighrem, ksour, e tighremt, kasbah, contrassegnati con il codice $\mathrm{K} 4 \mathrm{e}$ K5di Ifri; K23 K24 di El Hebs; e K21 K22 di Lakdima. Nella valle del Todhra a Tinghir, Kasbah Pasha, K 01 e ad Ait Mhamed la moschea diruta e tre kasbah, K04.

\section{Bibliography}

Ait El Haj, H. (2006). "Kasbahs et Ksour: un patrimoine en ruine”, in Espace Marocains, Janvier Février, pp. 27-40.

Bencherifa, A.; Popp, H. (1990). Le Maroc: es pace et société; actes du colloque maroco-allemand de Passau 1989, Passavia Universitätsverlag, Passau.

Gil Piqueras, M.T. (2014). Arquitectura de tierra en el Alto Atlas: Del Oasis de Mdagra al Valle del Outat, Tésis Doctoral, Universitat Politècnica de València, Valencia.

Golvin, L. (1957). Aspects de l'artisanat en Afrique du nord, Presses Universitaires de France, Paris.

Hensens, J. (1969). "Habitat rural traditionnel des oasis présahariennes: Le qsar problèmes de rénovation”, Bulletin Economique et Social du Maroc, 31, 114, pp. 83-107.

Jacques-Meunié, D. (1951). Greniers-citadelles au Maroc, Arts et Métiers Graphiques, Paris.

Lugan, B. (2000). "Les Berbères, la mémoire des sables", Voyages culturels et historiques de Clio, in http://www.clio.fr/BIBLIOTHEQUE/pdf/pdf_les_berberes_la_memoire_des_sables.pdf.

Majorelle, J. (1930). Les kasbahs de l'Atlas, Jules Meyniel, Paris.

Montagne, R. (1930). Villages et kasbas berbères: tableau de la vie sociale des berbères sédentaires dans le Sud du Maroc, Félix Alcan, París.

Naji, S. (2002). Art et Architectures Berbères du Maroc, Édisud Eddif, Aix-en-Provence, Casablanca.

Rodriguez-Navarro, P. (2014). "El destino de las kasbahs del Alto Atlas en Marruecos. Tres ejemplos en el valle del M'Goun", in Construcción con tierra, Patrimonio y Vivienda. X CIATTI, Universidad Valladolid, Cátedra Juan de Villanueva, Valladolid, pp. 167-176.

Terrasse H. (2010). Kasbas Berbéres, De l'Atlas et des oasis. Les grandes architectures du sud marocain, Actes Sud Ed., Arles. 